\title{
Status and Future Directions of Anti-metastatic Cancer Nanomedicines for the Inhibition of Cathepsin L
}

\author{
Tanveer A. Tabish ${ }^{1 \dagger}$, Md Zahidul I. Pranjol ${ }^{2 t}$, Jacqueline L. Whatmore ${ }^{3 *}$ and \\ Shaowei Zhang ${ }^{1 *}$
}

${ }^{1}$ College of Engineering, Mathematics and Physical Sciences, University of Exeter, Exeter, United Kingdom, ${ }^{2}$ School of Life Sciences, University of Sussex, Brighton, United Kingdom, ${ }^{3}$ Institute of Biomedical and Clinical Science, University of Exeter Medical School, Exeter, United Kingdom

OPEN ACCESS

Edited by:

Nagesh Kolishetti, Florida International University,

United States

Reviewed by:

Bapu Surnar

University of Miami Hospital,

United States

Dhivya R. Sudhan,

University of Texas Southwestern Medical Center, United States Olga Vasiljeva,

CytomX Therapeutics Inc.,

United States

*Correspondence:

Jacqueline L. Whatmore

j.l.whatmore@exeter.ac.uk

Shaowei Zhang

s.zhang@exeter.ac.uk

†These authors have contributed equally to this work

Specialty section:

This article was submitted to Biomedical Nanotechnology,

a section of the journal

Frontiers in Nanotechnology

Received: 17 December 2019

Accepted: 09 April 2020

Published: 06 May 2020

Citation:

Tabish TA, Pranjol MZI, Whatmore JL and Zhang S (2020) Status and Future

Directions of Anti-metastatic Cancer

Nanomedicines for the Inhibition of Cathepsin L. Front. Nanotechnol. 2:1.

doi: 10.3389/fnano.2020.00001
Angiogenesis, tissue invasion and metastasis in the tumour microenvironment are all critical hallmarks of cancer. Upregulation of cathepsin $L$ plays an important role in angiogenesis and metastasis through its ability to degrade the extracellular matrix, facilitating tissue remodeling and tumour cell invasion. Thus, cathepsin $L$ is a potential therapeutic target for anticancer nanomedicine, with its inhibition emerging as an innovative and potentially promising therapeutic intervention for the development of anti-invasion and anti-metastatic enzyme therapies. Nanotechnology-based platforms have been extensively tested in the anti-cancer nanomedicine field with effective anti-tumour efficacy. These nanodrugs can suppress tumour cell proliferation, thereby reducing tumour growth. Recently, nanomedicinal approaches have also emerged as effective anti-metastatic strategies, including the use of graphene oxide and gold nanoparticles. With a focus on recent advances in developing nanotechnology to inhibit cathepsin $L$, this review provides an in-depth examination of this stimulating field in the context of tumour microenvironments. Innovative anti-metastatic agents may lead to new options for the treatment of cancers.

Keywords: nanomedicine, graphene, nanopartcicles, cathepsin L, cancer

\section{INTRODUCTION}

Cancer treatment is challenging partly as a result of significant damage to adjacent healthy tissues, unwanted side-effects, high rate of recurrence, and associated costs. Novel approaches developed to reduce tumour resistance to chemo/immune/radio-therapy and the combination of these therapies have improved treatment efficacy. Tumour growth creates a specialized niche microenvironment which has been reported to include overexpression of cathepsin L (CathL) in some cancers. CathL is an ubiquitous cysteine proteinase that resides in the lysosomes of all cells; primarily, this enzyme breaks down endocytosed foreign proteins as well as misfolded/unfolded faulty protein quaternary structures in the lysosomes in acidic pH (Kirschke et al., 1977; Kominami et al., 1991). However, CathL has also been reported to be secreted out of the cell into the extracellular space (Kominami et al., 1991) and maybe active in the extracellular tumour microenvironment (Kirschke et al., 1977; Kominami et al., 1991). The development of innovative modalities for the inhibition of CathL could be an emerging anti-tumour therapy and recent work indicates a platform for investigating this. 
The emergence of nanomedicines provides the possibility to address the challenges associated with inhibition of CathL. Nanomaterials have emerged as effective anti-cancer agents in the areas of smart delivery and drug targeting for cancer diagnosis and treatment (Noureen et al., 2018b, 2019; Tabish et al., 2018b). The rapidly growing field of nanotechnology deals with structures less than $<100 \mathrm{~nm}$ in size (Tabish et al., 2018a), with these nanostructures exhibiting unique physiochemical characteristics which are different from their bulk counterparts. For the last three decades, nanomaterials have been extensively exploited for biomedical applications owing to their sizedependent properties (Noureen et al., 2018a). Recent studies have demonstrated that nanostructured graphenes, which are considered as a new and promising class of nanomedicine drugs due to their low toxicity, are significantly toxic to cancer cells (Tabish et al., 2018d). Graphene is a single atomic, 2D layer of $\mathrm{sp}^{2}$ hybridized carbon atoms covalently bonded in a honeycomb lattice (Tabish, 2018). Graphene offers many remarkable characteristics, for example high electron mobility, excellent mechanical strength, exceptionally good specific surface area, and good biocompatibility (Tabish et al., 2018c). We have recently reported the inhibition of cathepsin $\mathrm{D}$ and $\mathrm{L}$ using graphene oxide and explored the ability of enzymatic inhibition triggered by high surface area of graphene.

This review highlights the key role of CathL in cancer invasion, metastasis and angiogenesis in the context of its therapeutic potentials. This review additionally discusses the scientific evidence that nanostructured materials have antimetastatic features, and potential mechanisms of action for the inhibition of CathL.

\section{CATHEPSIN L}

Discovery of CathL was first reported as a major excreted protein from a transformed mouse fibroblast cell line and it was later identified as an important lysosomal enzyme (Kominami et al., 1988). It was thought at first that the precursor form of the enzyme (pCathL) was secreted from the cell and remained proteolytically inactive. However, reports have emerged that suggest that the extracellular precursor form of CathL remained enzymatically active at $\mathrm{pH}$ lower than 6.5 i.e., $\mathrm{pH}$ of the tumour microenvironment, although, it showed no activity at neutral $\mathrm{pH}$ (Kominami et al., 1988). This suggested that pCathL transportation bypassed recruitment to the lysosomes and instead, was secreted directly by exocytosis from the ER/Golgi pathway, with an ECM-degradation role in the tumour microenvironment. Interestingly, the mature form of CathL (proteolytically active) was also found in the extracellular space both in physiology and disease. For instance, CathL was shown to degrade extracellular membrane proteins and play an important role in bone remodeling through extracellular proteolytic activity (Mason et al., 1986; Maciewicz et al., 1990; Nguyen et al., 1990; Maciewicz and Wotton, 1991; Ishidoh and Kominami, 1995; Nosaka et al., 1999). Recently, recombinant mature CathL was shown to stimulate axonal growth in a proteolytic-independent manner (Tohda and Tohda, 2017). Secreted CathL has also been shown to be protective against bacterial infection in mice airways (Xu et al., 2013).

CathL is processed via the default ER/Golgi pathway where preprocathepsin L is cleaved into procathepsin L (pCathL). pCathL can either consist of a single chain of molecular mass of $30 \mathrm{kDa}$ and/or a two-chain form with molecular masses 25 and 5 $\mathrm{kDa}$ (Kominami et al., 1988; Nishimura et al., 1988). The protein is then transported in newly formed vesicles to endosomes, and via an interaction with the mannose-6-phosphate/receptor (M6P/M6PR), the pCathL enters the lysosome (Dong and Sahagian, 1990). The N-linked oligosaccharide mannose is covalently attached to CathL and undergoes phosphorylation by phosphoesterases in the cis Golgi and becomes M6P (Lang et al., 1984). A resident M6PR protein in the trans Golgi network interacts with these M6P groups which facilitates the delivery of the protein to lysosomes (Kornfeld, 1986). On their arrival, the acidic $\mathrm{pH}$ of the lysosomes initiates dissociation of the cathepsins from the receptors, resulting in the removal of the phosphate group by lysosomal acid phosphatase (Samie and Xu, 2014).

The enzyme inhibitors 1, and 10-phenanthrolin and pepstatin induced partial inhibition of the processing of pCathL to its mature form. This observation suggested that the active form of CathL in the lysosomes is generated via proteolytic cleavage by metalloproteinases or aspartic proteases such as CathD (Ritonja et al., 1988; Ishidoh et al., 1991). At the very low $\mathrm{pH}$ of the lysosome ( $\mathrm{pH} \mathrm{3}$ ), pCathL can also be autoprocessed to form mature CathL which is highly active at $\mathrm{pH} 5.5$ (Mason et al., 1987; Mason and Massey, 1992). For instance, in physiology, the Ii peptide of the MHC II complex is degraded by active CathL, allowing degraded peptides of foreign or self-proteins to be presented to stimulate an immune response (Nakagawa et al., 1998; Hsieh et al., 2002). Indeed, mice deficient in CathL were observed to have reduced CD4+ T cells. Intracellular CathL has been reported to play a homeostatic role in the epidermis and regular hair follicle morphogenesis and cycling. For instance, mice with CathL deficiency suffer from periodic hair loss and epidermal hyperplasia, acanthosis, and hyperkeratosis (Roth et al., 2000).

Interestingly, secreted CathL retains its function as a protease and plays a key role in a range of functions. For instance, a role of CathL in physiological bone remodeling has been described where a significant decrease in bone volume in trabecular bone, but not cortical, could be observed in CathL-null mice compared to wild type (Potts et al., 2004). Estrogen-induced secreted CathL was suggested to regulate bone turnover during normal development and in pathologies (Potts et al., 2004). Basic fibroblast growth factor (bFGF) was shown to induce an upregulation of CathL mRNA and its release. It is also believed that, in a hypoxic, acidic environment, cells increase production of lysosomal enzymes such as CathL and CathD which results in their hypersecretion (Cuvier et al., 1997). However, the exact mechanism of CathL secretion still remains unanswered. CathL has a relatively low affinity for M6PR (von Figura and Hasilik, 1986; Dahms et al., 1989; Dong et al., 1989), thus, it is believed that only some binds to the available M6PR and the rest is secreted out of the cells via conventional protein trafficking (Noureen et al., 2019). An overexpression of CathL at its protein 
level, saturation of $\mathrm{M} 6 \mathrm{PR}$, and downregulation or redistribution of this enzyme to the plasma membrane has also been suggested (Achkar et al., 1990; Prence et al., 1990). Nonetheless, secreted CathL is now understood to play a role in normal physiological processes and disease.

\section{CATHEPSIN L IN CANCER}

The homeostasis of the tumour microenvironment is dysregulated compared to normal tissue physiology. As a solid tumour grows, the cells within it secrete numerous enzymes and pro-survival factors such as vascular endothelial growth factor (VEGF), bFGF, inflammatory cytokines, ECM remodeling enzymes and proteins, creating a proinflammatory environment (Hanahan and Weinberg, 2011). These secreted proteins create a cross-talk between local cells, stimulating them to behave in favor of the tumour growth (Figure 1). For instance, cancer-associated fibroblasts (CAFs) increase secretion of ECM proteins as well as pro-tumourigenic factors in the tumour microenvironment (De Palma et al., 2017). Secretion of major enzymes such as MMPs and LOX1 degrade and remodel the local ECM allowing tumour cells to invade the host organ and endothelial cells from the local microvasculature to form new blood vessels, in a process called angiogenesis (De Palma et al., 2017).

In the last 3 decades, cysteine cathepsins, particularly CathL, have been found to play a role in cancer progression, although this enzyme remains less well-studied than other cathepsins. An enzymatic role for CathL has been reported in tumour invasion and metastasis, mainly by degrading bone and several ECM components (von Figura and Hasilik, 1986; Dahms et al., 1989; Dong et al., 1989; Cuvier et al., 1997; Roth et al., 2000; Potts et al., 2004). For instance, breast cancer cells overexpress CathL, which induces bone degradation in breast and prostate cancer metastasis (Qin et al., 2016; Sudhan et al., 2016). In prostate cancer patients with skeletal metastasis, tumour cells have been shown to alter cytokine milieu in the microenvironment, resulting in activation of osteoclasts and selective upregulation of osteoclastic CathL production and secretion (Leto et al., 2010, 2016; Sudhan et al., 2016). Activated osteoclasts increase bone resorption, releasing protumourigenic growth factors. This, along with tumour cellsecreted CathL, results in an increased degradation of bone matrices that supports aggressive growth of metastases (Yoneda and Hiraga, 2005). In immortalized fibroblasts (NIH/3T3 cells), CathL-overexpression was linked to metastasis following RAS

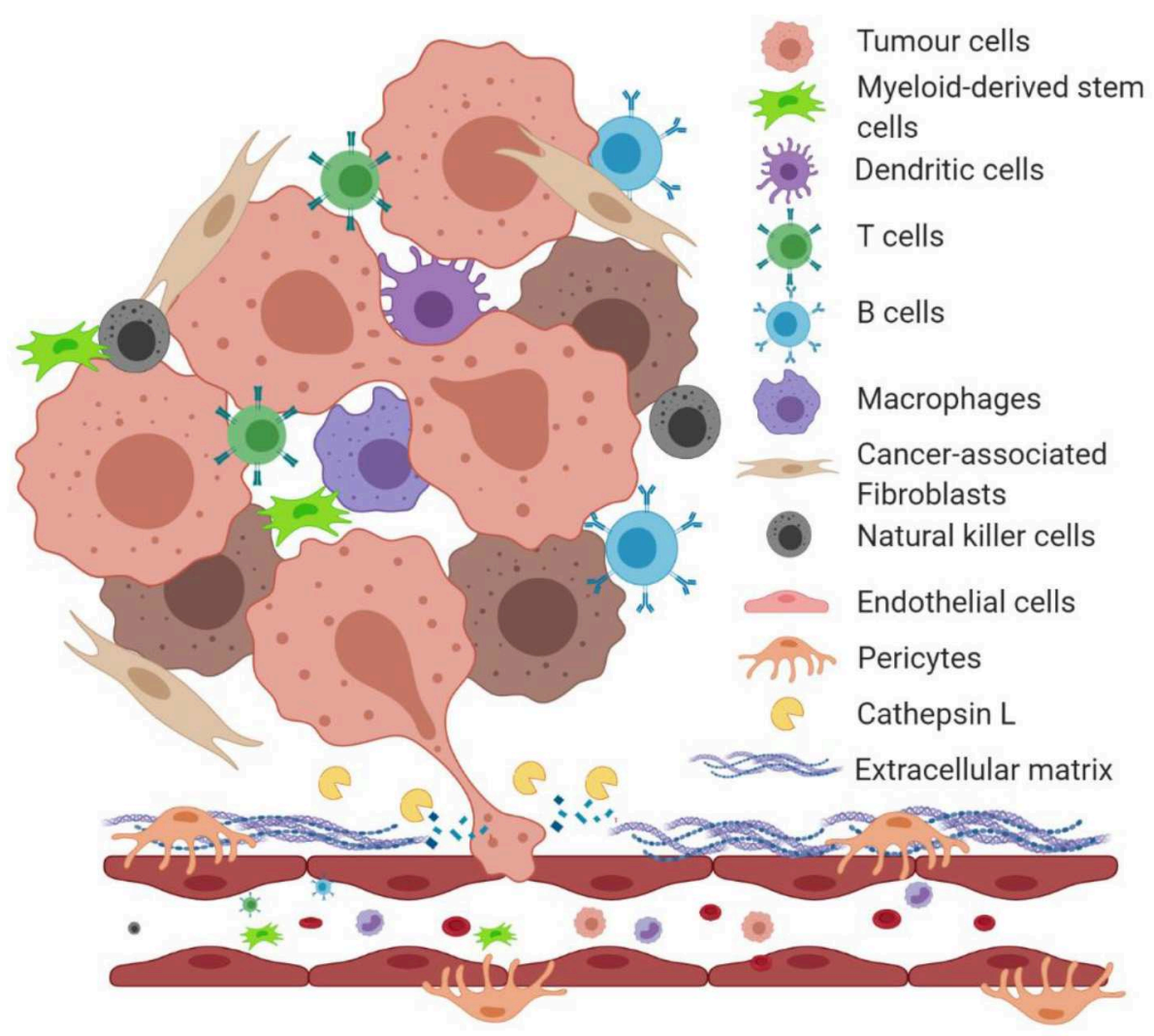

FIGURE 1 | An illustration of the tumour microenvironment and a role of proteolytically active cathepsin $L$ in degrading extracellular matrix proteins. Under the hypoxic condition of the tumour microenvironment, growing tumours secrete factors that induce a cross-talk between surrounding stromal cells. Tumour cells and cancer-associated fibroblasts secrete extracellular matrix-degrading enzymes such as cathepsin L (CathL) that catalyze the breakdown of the local basement membrane, allowing tumour cells to migrate and "squeeze" through the disrupted ECM and enter the local circulation to metastasize to distant organs. 
transformation in vitro (Chambers et al., 1992; Sui et al., 2016), suggesting a role in the transformation of non-cancerous cells to cancerous ones. This was further shown in melanoma cells whereby overexpression of CathL was shown to be responsible for inducing a metastatic state in these cells in vitro (Frade et al., 1998). An extra-lysosomal role for CathL was also reported in human and murine melanoma cells in the context of metastasis (Ishidoh and Kominami, 1998). In melanoma cells (B16F10), CathL-induced cellular migration is thought to be partly responsible for invasion (Yang and Cox, 2007). For instance, a $70 \%$ reduction in invasion and migration was observed in clones transfected with CathL antisense compared with control. CathL-induced migration in melanoma cells was confirmed by the observation that anti-CathL antibody mediated a marked inhibition of the pro-migratory effect (Rousselet et al., 2004). However, CathL had no effect on cellular proliferation (Yang and Cox, 2007). CathL-induced pancreatic cancer cell invasion was also observed in mice. In mice models carrying pancreatic tumour, CathL was reported to degrade the extracellular Nterminal domain of E-cadherin which acts as an adhesion molecule between adjacent cells (Gocheva et al., 2006). This degradation was suggested to result in an increase in cancer cell dissemination and invasion (Gocheva et al., 2006). However, interestingly, a $58 \%$ decrease in proliferation was also observed in these CathL knockout cells, although the exact mechanism of action, i.e., proteolytic or non-proteolytic, was not investigated (Gocheva et al., 2006).

Hypersecretion of CathL has been observed in the sera of malignant epithelial ovarian cancer (EOC) patients (Nishida et al., 1995; Zhang et al., 2014), possibly due to the overexpression of this enzyme in tumour cells within the hypoxic microenvironment, which was shown by an upregulated expression of CathL mRNA levels in tumours. This was later reported to play an important role in the invasion and metastasis of EOC, and hence was suggested to be a marker for advanced stage ovarian cancer (Zhang et al., 2014). In our immunohistochemical studies, we observed significantly increased CathL expression in the endothelium of vessels within the omentum hosting secondary tumour foci in patients suffering from high-grade serous carcinoma compared with control patients with benign ovarian cystadenoma (Winiarski et al., 2014). The omental fat is highly vascularized (Platell et al., 2000) and contains a vast amount of ECM proteins such as collagen (Alkhouli et al., 2013). Thus, in the inflamed, hypoxic tumour microenvironment, tumour cells may induce overexpression and hypersecretion of proteolytically active CathL, among other enzymes, from surrounding cells that in turn degrade local basement membrane allowing tumour cell invasion of the omentum. Interestingly, when investigated for a direct, non-proteolytic role, CathL was observed to have less influence on proliferation of the A2780 ovarian cancer cell line (Zhang et al., 2014). However, the proliferative and the invasive capacity of SKOV3 ovarian cancer cells was significantly inhibited by inducing downregulation of CathL-gene expression (Zhang et al., 2015).

\section{CATHEPSIN L IN ANGIOGENESIS}

A presence of CathL in the extracellular space within the microenvironment, along with its role in ECM degradation suggests a potential role in inducing angiogenesis i.e., allowing migration of ECs through digested ECM toward tumour foci (Figure 2). However, studies using different experimental model systems suggested both pro-and anti-angiogenic roles for CathL. For instance, bFGF-transfected skeletal muscle cells demonstrated an increased secretion of CathL which induced migration in human umbilical vascular ECs (HUVECs) in vitro (Chung et al., 2011). This migratory effect was assessed in the presence of Z-Phe-Tyr-Cho, an inhibitor of CathLenzymatic activity, which resulted in a significant reduction in HUVEC migration, suggesting a proteolytic-dependent mechanism. Interestingly, in the same study, CathL induced cJun N-terminal kinase (JNK) activation in cellular migration, possibly via cleaving and releasing a growth factor that acted on a specific cell surface receptor upstream, although the authors did not explore this in the study (Chung et al., 2011).

We reported secretion of CathL by EOC cells (SKOV3 and A2780) in vitro (Winiarski et al., 2013), and more recently showed that exogenous CathL induced proliferation, migration and angiogenic tube formation of human omental microvascular endothelial cells (HOMECs) by activating the ERK1/2 pathway in a proteolytic-independent manner (Pranjol et al., 2019a). A potent and selective inhibitor (FY-CHO) of CathL-proteolytic activity was utilized to assess whether CathL remained proteolytically active at $\mathrm{pH}$ 7. FY-CHO is known to prevent secreted CathL-induced bone resorption in unfractionated rat bone cells in the pit formation assay and reduce bone-weight loss in mice in a dose-dependent manner (Woo et al., 1996). Moreover, studies investigating breast and prostate cancer reported this inhibitor as a potent compound that successfully inhibited CathL mediated migration and invasion in vitro (Sudhan and Siemann, 2013). We found that CathL remained proteolytically active at the $\mathrm{pH}$ of cell culture media (i.e., $\mathrm{pH}$ 7.2-7.4), however, the inhibitor did not induce a reduction in CathL-induced cellular proliferation (Pranjol et al., 2019a). Thus, it was concluded that CathL induces HOMEC proliferation via a mechanism that is independent of its proteolytic activity, probably via an allosteric interaction with an unknown receptor, which remains to be investigated.

In an attempt to further investigate the extracellular role of CathL, our group has recently demonstrated that the enzyme induces an upregulation of galectin-1, a lectin protein, at both the mRNA level and secreted protein level in HOMECs (Pranjol et al., 2019b). Galectin-1 is known to play various roles in cell biology including cell proliferation and migration in cancer (van den Brûle et al., 2003; Croci et al., 2012; D’Haene et al., 2013; Hsu et al., 2013). We found that CathL-induced secreted galectin-1 further promotes angiogenesis via increasing proliferation and migration of HOMECs in an autocrine manner. Thus, CathL not only directly induces proangiogenic changes in ECs, but also acts indirectly to contribute to tumour angiogenesis in cancer metastasis. 


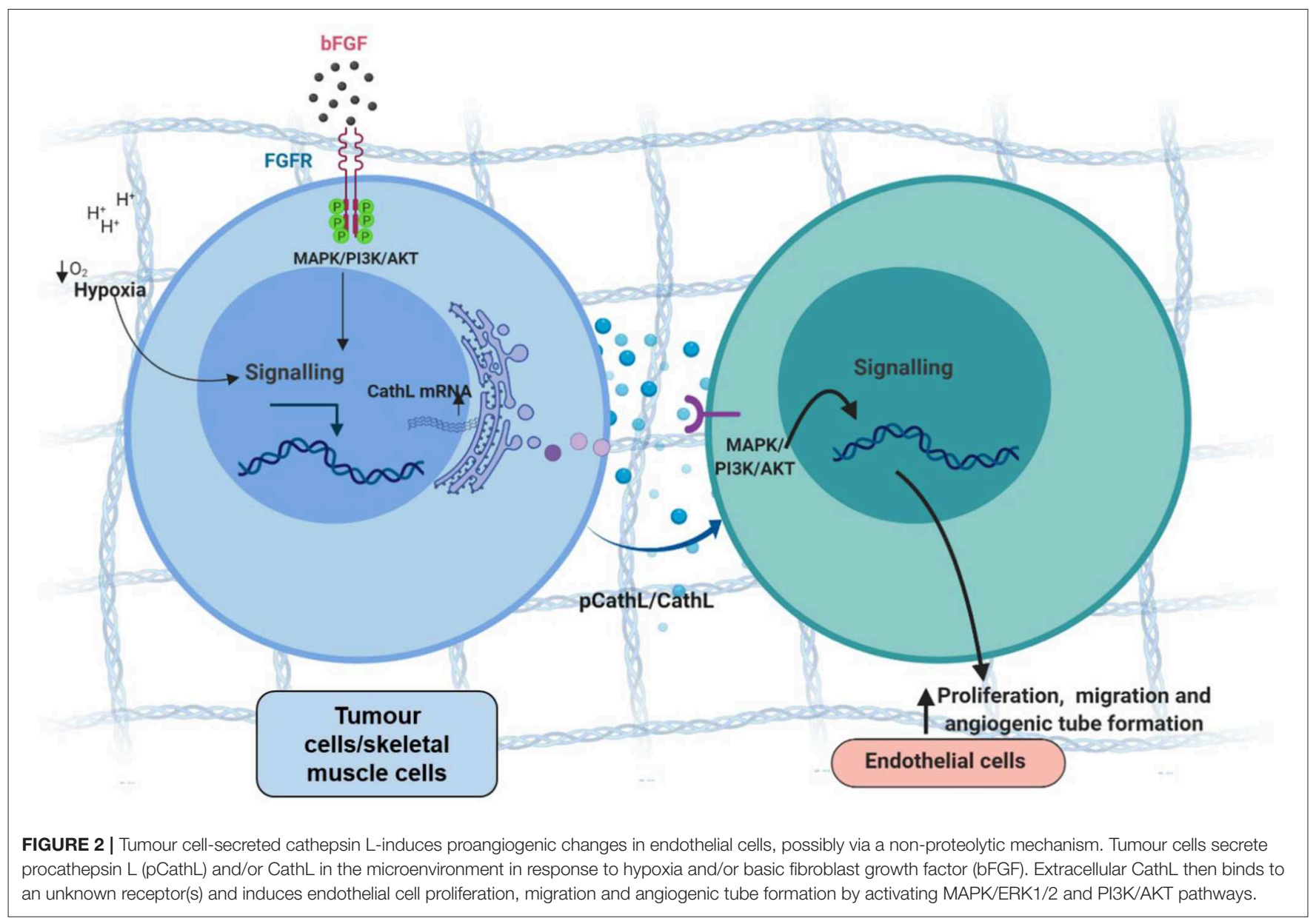

In contrast, there are studies that disagree with the aforementioned proangiogenic effect of CathL. For instance, release of endostatin, a potent inhibitor of angiogenesis, was reported to be released by CathL-mediated cleavage of ECM collagen (Felbor et al., 2000). The slightly acidic tumour microenvironment favors this anti-angiogenic function of CathL. In other studies, CathL was found to have no effect on ECs (Gocheva et al., 2006). For instance, in CathL-knockout RT2mice (RT2, or RIP1-Tag2 mice carry the SV40 large T-antigen under the insulin promoter II), no change in microvascular density was observed in cancerous lesions (pancreatic cancer), compared with control mice, suggesting that angiogenic switching is not controlled by CathL (Gocheva et al., 2006). Interestingly, CathB and CathS were found to be very significant in inducing angiogenesis in the same study (Gocheva et al., 2006). Nevertheless, CathL has been reported elsewhere to play a major proangiogenic role. For instance, mice with CathL deficiency suffered from impaired neovascularization. Moreover, when CathL-deficient bone marrow cells were injected into mice, a significant reduction in angiogenesis was observed, suggesting that CathL plays a critical role in endothelial progenitor cell (EPC) mediated neovascularization (Urbich et al., 2005). This was further supported by Shimada and colleagues, who noted that
EPC-expressed CathL was vital in intraocular angiogenesis (Shimada et al., 2010).

A pro-tumourigenic and pro-angiogenic role of CathL signifies a window of opportunity in developing anticancerous therapies. A number of approaches such as inhibitor development, nano-based CathL targeting, have been an interest in the recent years. For example, Gocheva et al. utilized panspecific cathepsin protease inhibitor in studying pancreatic cancer progression (Gocheva et al., 2006). However, the inhibitor had no significant effect on tumour growth and angiogenesis (Gocheva et al., 2006). This may have been because the enzyme was also acting in a non-proteolytic manner, as we have shown in our studies (Pranjol et al., 2019a). Thus, new strategies, including nanotechnology based targeting could be researched further.

\section{CATHEPSIN L INHIBITION}

Mallari et al. (2008) studied the effects of thiosemicarbazones against the enzymatic activity of human and trypanosomal cathepsins ( $\mathrm{B}$ and $\mathrm{L}$ ) and evaluated the toxicity of these compounds against four cell lines (BJ, Raji, HEK 293, and HEP G2), revealing low toxicity and good membrane permeability. Kevin Pinney's research group have synthesized astructurally and 
biologically relevant anti-tumour series of thiosemicarbazonebased inhibitors of CathL. These agents incorporate a diverse range of electrophilic moieties to breakdown the structure of CathL via electrostatic attraction forces. In one study, they prepared thirty six functionalized benzophenone thiosemicarbazone compounds to assess the inhibition of CathL: the efficient inhibitors of CathL (IC50 $<85 \mathrm{nM}$ ) among these compunds were six which combined a bromo group in an aryl ring in addition to other functionalities involved within the second aryl ring. These active compounds also revealed good results of cell invasion inhibition against a human prostate cancer cell line (DU-145) (Kishore Kumar et al., 2010). Another study (Parker et al., 2015) reported the production and assessment of benzoylbenzophenone thiosemicarbazone compounds to inhibit the CathL. Three compounds demonstrated highly efficient inhibition. This research group also fabricated a library of 36 compounds based on aryl-alkyl ring compounds (which contain nitrogen and carbon atoms). Furthermore, this work also included the functionalization based on oxygen, sulfur, sulfoxide, and sulfone groups for investigating the morphology and inhibition linked to these compounds and their potency for CathL activity. Among these thirty six compounds, five were highly active against CathL (Song et al., 2013). Parker et al. (2017) reported the KGP94, a member of the group of functionalized benzophenone thiosemicarbazone analogs, which showed similar inhibition capacity for CathL. However, this compound has low water dispersibility and to improve aqueous solubility, the phosphate prodrug, KGP420 can be used which converts to the parent compound, KGP94. Chavarria et al. (2012) also reported that KGP94 inhibits CathL. KGP94 significantly improved the inhibition of CathL. This is also the first in vitro study on the inhibition of CathL where growth retardation was achieved against a mouse carcinoma cell line $\mathrm{C} 3 \mathrm{H}$. These promising benzophenone and thiochromanone compounds have emerged as promising CathL inhibitors but their use is limited by complex chemical configuration and synthesis routes.

\section{NANOTECHNOLOGY-BASED ADVANCES IN CATHEPSIN L INHIBITION}

The anionic properties, surface charge, high surface area and small diameter of nanomaterials make them ideal candidates for the degradation and inhibition of a variety of enzymes. Recent findings have described the effect of size, shape, and functionalization of nanoparticles for the inhibition of the cathepsin family (Sée et al., 2009; Speshock et al., 2011; Tabish et al., 2019). Unfortunately, very little is known about the mechanism of nanoparticle-cathepsin interaction. A deep understanding of the biochemical interactions of nanoparticles with cathepsin at molecular and intra-, sub-cellular levels is, therefore, crucial for the development, testing and utilization of nanoparticles as nanodrugs to inhibit cathepsin. Sée et al. (2009) have demonstrated that functionalized gold nanoparticles (with peptides and proteins) have the potential to be degraded during cellular localization within endosomal compartments as entry pathways. The potential mechanism of this process occurs via peptide cleavage by the protease CathL. This work was focussed on the cellular uptake and enzymatic alterations of gold nanostructures against Hela, SK-N-AS, D-283 Med, MEF, and DAOY cell lines. This study has not discussed the inhibition of CathL and nanoparticle-CathL interaction at molecular or cellular levels. Speshock et al. (2011) reported gold (10 nm of size) and silver (10 and $30 \mathrm{~nm}$ of size) nanoparticles and investigated the effects of these nanoparticles on CathL activity, revealing that gold nanoparticles stimulated CathL activity in cells but not silver nanoparticles. These results confirmed that functional groups on the surface of gold nanoparticles could potentially have been lost upon cell internalization and localization in Vero cells, although this work did not provide any evidence of cellular internalization and localization of nanoparticles which largely remains unexplored and unknown. The high levels of internalization and cellular uptake of AuNPs stimulated CathL activity.

Both studies mentioned above showed the cellular activity of CathL using gold nanoparticles. Despite recent advancements in using gold nanostructures for optical diagnostics (such as Raman scattering systems, photoacoustic imaging modality) and therapeutics (such as photothermal therapy), understanding the ultimate fate, clearance, excretion and biodistribution of these nanostructures in living systems is a major knowledge gap in their use as theranostic agents. Importantly, bioinformatics, pharmacokinetics and pharmacodynamics are important tools to further uncover the mechanistic phenomena of nanoparticlecathepsin interaction. Furthermore, inhibition of the cathepsin family using nanotechnology-based approaches is a major knowledge gap. Our group has recently shown (Tabish et al., 2019) that highly biocompatible graphene oxide could be used as a strong inhibitor for CathL activity in a time, dose and $\mathrm{pH}$ dependent manner. Different analytical tools such as Raman scattering system, Fourier Transform Infrared Spectroscopy (FTIR), water contact angles and surface energy revealed the strong bonding and adsorption mechanisms of this inhibition activity against CathL. This was the first study to examine the inhibition of CathL using nanomaterials under biologically and clinically relevant conditions e.g., appropriate $\mathrm{pH}$. The method of graphene oxide synthesis described in this work was very straightforward, cost-effective and highly biocompatible and showed the potential for development of nanomedicine-based anti-metastatic agents to inhibit of cell invasion. In this work, the batch adsorption process was adopted to quantity the effect of $\mathrm{pH}$ on the inhibition of CathL using graphene oxide. CathL adsorption was revealed to be $\mathrm{pH}$ - and concentration-dependent, representing that the highest adsorbed quantities were at the acidic $\mathrm{pH}$ of 5 . The adsorption capacity of graphene oxide at the concentration of $1000 \mu \mathrm{g} / \mathrm{mL}$ was higher than $90 \%$ after $20 \mathrm{~min}$. In a recently published study, Junior et al. (2019) encapsulated cysteine protease inhibitors (Neq0551, Neq0554, and Neq0568) into apoferritin protein nanocages and seeded them with two cancer cacell lines (HCT-116 colorectal carcinoma cells and MiaPaCa-2 pancreatic adenocarcinoma cells) to investigate the inhibition of CathL. In both cells, CathL activity was efficiently inhibited with nanoagents (at the concentrations above $25 \mu \mathrm{M}$ ), in comparison to pristine inhibitor groups, potentially by 
retaining intracellular/lysosomal nanoagents for a greater period of time. Recently Bratovš et al. (2019) developed a liposomal drug carrier system conjugated with a small endogenous protein inhibitor of cathepsins, stefin A [D61C], as the targeting moiety and demonstrated that stefin A-conjugated liposomes could inhibit CathL in vitro and in vivo (mice) models. This approach could potentially be used as diagnostic tool and also for encapsulation of chemotherapeutic drugs because stefin has large protease-binding region, high thermostability and lack of disulphide bonds and high affinity and high specificity toward its endogenous targets (Jenko et al., 2003; Renko et al., 2010; Mikhaylov et al., 2014).

In general, covalent inhibitors are considered as potential inhibitors for CathL with reduced collateral side effects. However, these small compounds and inhibitors suffer from off-target toxic effects and limited reactive groups existing onto their surfaces. Therefore, novel design and development of these compounds is significantly important to facilitate the high affinity toward the target enzyme. An alternative approach to modify these small compounds is to use nanoparticles as core structures. Although toxicity associated with these small compounds can be minimized by using nanomaterials. Nanomaterials are already available in clinical settings for selective and targeted delivery of drugs and photochemicals for disease management (Ronkainen and Okon, 2014; Patra et al., 2018). Small inhibitor compounds can be encapsulated onto the core of biocompatible nanoparticles for selective and targeted delivery to the site as well as selective inhibition of CathL. The efficient design of nanomaterials and their payloads provides beneficial structures that allow efficient inhibition of CathL. Selective inhibition of CathL is a promising strategy toward the efficient designs of anti-metastatic agents, which still remains an urgent unmet need. Although, drugs for the inhibition of cathepsin $\mathrm{K}, \mathrm{B}, \mathrm{C}$, and S have extensively been studied (Withana et al., 2012; Sudhan and Siemann, 2015; Flanagan-Steet et al., 2018; Lu et al., 2018; Nwosu et al., 2018; Panwar et al., 2018; Xue et al., 2019) there has been limited success with CathL.

\section{SUMMARY AND FUTURE OUTLOOK}

High levels of CathL in tumours have been linked with poor prognosis in breast, ovarian, prostate, brain, and lung cancers. High biocompatibility and low cytotoxicity are the most significant therapeutic features of nanomedicines required of anti-invasion and anti-metastatic agents to suppress tumour

\section{REFERENCES}

Achkar, C., Gong, Q. M., Frankfater, A., and Bajkowski, A. S. (1990). Differences in targeting and secretion of cathepsins $\mathrm{B}$ and $\mathrm{L}$ by BALB/3T3 fibroblasts and Moloney murine sarcoma virus-transformed BALB/3T3 fibroblasts. J. Biol. Chem. 265, 13650-13654.

Alkhouli, N., Mansfield, J., Green, E., Bell, J., Knight, B., and Liversedge, N., et al. (2013). The mechanical properties of human adipose tissues and their relationships to the structure and composition of the microenvironment-enhanced metastasis (Tabish et al., 2017; Fan et al., 2018; Zhang et al., 2020). To date, the most promising nanomedicine-based anti-metastatic agents are gold nanoparticles and nanostructured graphene. Tunable size, physiochemical and shape-dependent surface properties, $\mathrm{pH}$ dependent surface charge, high specific surface area, luminescent characteristics and the ability for selective and targeted delivery of these nanoparticles make them ideal candidates as anti-metastatic agents. These agents show a high ability to inhibit and breakdown CathL, which is desired for the cell invasion. Both gold and graphene nanostructures have widely been demonstrated for cancer treatment in preclinical studies and are considered as promising candidates for therapeutic interventions. Recent studies on the high efficiency of nanomaterials for the inhibition of CathL and other cathepsin family members could potentially revolutionize the emerging field of nanomedicine.

It has been reported that nanostructured materials have antiproliferative, selective and targeted light-mediated therapeutic activities. Therefore, nanomaterials-based anti-metastatic agents could complement other therapies such as antiproliferative, photodynamic and photothermal therapies. However, the molecular mechanistic insights are yet to be fully elucidated in this field of anti-metastatic therapy. Critical assessment of optimum anti-metastatic activities of nanomaterials, therefore, warrants further studies at in-vitro and in-vivo levels. The combination of these nanoagents with clinically available drugs may offer a new broad-spectrum impairment of tumour cell function, and it is hoped that these nanodrugs have the therapeutic potential to become new and improved therapeutic modalities.

\section{AUTHOR CONTRIBUTIONS}

TT and MP wrote this paper. JW and SZ supervised this work and revised the manuscript. All authors read and approved the manuscript.

\section{FUNDING}

This work was supported by EPSRC Center for Doctoral Training in Metamaterials, XM2 (Grant no. EP/L015331/1) the University Of Exeter EX4, United Kingdom, and FORCE Cancer Charity (Grant No. 50703) United Kingdom. 
Chavarria, G. E., Horsman, M. R., Arispe, W. M., Kumar, G. K., Chen, S. E., Strecker, T. E., et al. (2012). Initial evaluation of the antitumour activity of KGP94, a functionalized benzophenone thiosemicarbazone inhibitor of cathepsin L. Eur. J. Med. Chem. 58, 568-572. doi: 10.1016/j.ejmech.2012. 10.039

Chung, J. H., Im, E. K., Jin, T. W., Lee, S. M., Kim, S. H., and Choi, E. Y., et al. (2011). Cathepsin L derived from skeletal muscle cells transfected with bFGF promotes endothelial cell migration. Exp Mol Med. 43, 179-188. doi: 10.3858/emm.2011.43.4.022

Croci, D. O., Salatino, M., Rubinstein, N., Cerliani, J. P., Cavallin, L. E., and Leung, H. J., et al. (2012). Disrupting galectin-1 interactions with N-glycans suppresses hypoxia-driven angiogenesis and tumourigenesis in Kaposi's sarcoma. J. Exp. Med. 209, 1985-2000. doi: 10.1084/jem.20111665

Cuvier, C., Jang, A., and Hill, R. P. (1997). Exposure to hypoxia, glucose starvation and acidosis: effect on invasive capacity of murine tumour cells and correlation with cathepsin $(\mathrm{L}+\mathrm{B})$ secretion. Clin. Exp. Metast. 15, 19-25. doi: 10.1023/A:1018428105463

Dahms, N. M., Lobel, P., and Kornfeld, S. (1989). Mannose 6-phosphate receptors and lysosomal enzyme targeting. J. Biol. Chem. 264, 12115-12118.

De Palma, M., Biziato, D., and Petrova, T. V. (2017). Microenvironmental regulation of tumour angiogenesis. Nat. Rev. Cancer 17, 457-474. doi: $10.1038 /$ nrc.2017.51

D'Haene, N., Sauvage, S., Maris, C., Adanja, I., Le Mercier, M., and Decaestecker, C., et al. (2013). VEGFR1 and VEGFR2 involvement in extracellular galectin-1- and galectin-3-induced angiogenesis. PLOS ONE 8:e67029. doi: 10.1371/journal.pone.0067029

Dong, J. M., Prence, E. M., and Sahagian, G. G. (1989). Mechanism for selective secretion of a lysosomal protease by transformed mouse fibroblasts. J. Biol. Chem. 264, 7377-7383

Dong, J. M., and Sahagian, G. G. (1990). Basis for low affinity binding of a lysosomal cysteine protease to the cation-independent mannose 6-phosphate receptor. J. Biol. Chem. 265, 4210-4217.

Fan, W., Zhang, W., Alshehri, S., and Garrison, J. C. (2018). Increasing time on target: utilization of inhibitors of cysteine cathepsins to enhance the tumour retention of receptor-targeted agents. Chem. Commun. 54, 11268-11271. doi: 10.1039/C8CC05982A

Felbor, U., Dreier, L., Bryant, R. A., Ploegh, H. L., Olsen, B. R., and Mothes, W. (2000). Secreted cathepsin L generates endostatin from collagen XVIII. EMBO J. 19, 1187-1194. doi: 10.1093/emboj/19.6.1187

Flanagan-Steet, H., Christian, C., Lu, P. N., Aarnio-Peterson, M., Sanman, L., Archer-Hartmann, S., et al. (2018). TGF- $B$ regulates cathepsin activation during normal and pathogenic development. Cell Rep. 22, 2964-2977. doi: 10.1016/j.celrep.2018.02.066

Frade, R., Rodrigues-Lima, F., Huang, S., Xie, K., Guillaume, N., and BarEli, M. (1998). Procathepsin-L, a proteinase that cleaves human C3 (the third component of complement), confers high tumourigenic and metastatic properties to human melanoma cells. Cancer Res. 58, 2733-2736. doi: 10.1016/S0161-5890(98)90609-5

Gocheva, V., Zeng, W., Ke, D., Klimstra, D., Reinheckel, T., and Peters, C., et al. (2006). Distinct roles for cysteine cathepsin genes in multistage tumourigenesis. Genes Dev. 20, 543-556. doi: 10.1101/gad.1407406

Hanahan, D., and Weinberg, R. A. (2011). Hallmarks of cancer: the next generation. Cell 144, 646-674. doi: 10.1016/j.cell.2011.02.013

Hsieh, C. S., deRoos, P., Honey, K., Beers, C., and Rudensky, A. Y. (2002). A role for cathepsin $\mathrm{L}$ and cathepsin $\mathrm{S}$ in peptide generation for MHC class II presentation. J. Immunol. 168, 2618-2625. doi: 10.4049/jimmunol.168.6.2618

Hsu, Y. L., Wu, C. Y., Hung, J. Y., Lin, Y. S., Huang, M. S., and Kuo, P. L. (2013). Galectin-1 promotes lung cancer tumour metastasis by potentiating integrin alpha6beta 4 and Notch1/Jagged 2 signaling pathway. Carcinogenesis 34 , 1370-1381. doi: 10.1093/carcin/bgt040

Ishidoh, K., and Kominami, E. (1995). Procathepsin L degrades extracellular matrix proteins in the presence of glycosaminoglycans in vitro. Biochem. Biophys. Res. Commun. 217, 624-631. doi: 10.1006/bbrc.1995.2820

Ishidoh, K., and Kominami, E. (1998). Gene regulation and extracellular functions of procathepsin L. Biol. Chem. 379, 131-135.

Ishidoh, K., Muno, D., Sato, N., and Kominami, E. (1991). Molecular cloning of cDNA for rat cathepsin C. Cathepsin C, a cysteine proteinase with an extremely long propeptide. J. Biol. Chem. 266, 16312-16317.
Jenko, S., Dolenc, I., Gunčar, G., Doberšek, A., Podobnik, M., and Turk, D. (2003). Crystal structure of Stefin A in complex with cathepsin $\mathrm{H}$ : N-terminal residues of inhibitors can adapt to the active sites of endo-and exopeptidases. J. Mol. Biol. 326, 875-885. doi: 10.1016/S0022-2836(02)01432-8

Junior, J. C. Q., Carlos, F. D. R. R., Montanari, A., Leitão, A., Mignone, V. W., Arruda, M. A., et al. (2019). Apoferritin encapsulation of cysteine protease inhibitors for cathepsin L inhibition in cancer cells. RSC Adv. 9, 36699-36706. doi: 10.1039/C9RA07161J

Kirschke, H., Langner, J., Wiederanders, B., Ansorge, S., and Bohley, P. (1977). Cathepsin, L. A new proteinase from rat-liver lysosomes. Eur. J. Biochem. FEBS 74, 293-301. doi: 10.1111/j.1432-1033.1977.tb11393.x

Kishore Kumar, G. D., Chavarria, G. E., Charlton-Sevcik, A. K., Arispe, W. M., Macdonough, M. T., Strecker, T. E., et al. (2010). Design, synthesis, and biological evaluation of potent thiosemicarbazone based cathepsin L inhibitors. Bioorg. Med. Chem. Lett. 20, 1415-1419. doi: 10.1016/j.bmcl.2009.12.090

Kominami, E., Tsukahara, T., Hara, K., and Katunuma, N. (1988). Biosyntheses and processing of lysosomal cysteine proteinases in rat macrophages. FEBS Lett. 231, 225-228. doi: 10.1016/0014-5793(88)80736-1

Kominami, E., Ueno, T., Muno, D., and Katunuma, N. (1991). The selective role of cathepsins B and D in the lysosomal degradation of endogenous and exogenous proteins. FEBS Lett. 287, 189-192. doi: 10.1016/0014-5793(91)80048-8

Kornfeld, S. (1986). Trafficking of lysosomal enzymes in normal and disease states. J. Clin. Invest. 77, 1-6. doi: 10.1172/JCI112262

Lang, L., Reitman, M., Tang, J., Roberts, R. M., and Kornfeld, S. (1984). Lysosomal enzyme phosphorylation. Recognition of a protein-dependent determinant allows specific phosphorylation of oligosaccharides present on lysosomal enzymes. J. Biol. Chem. 259, 14663-14671.

Leto, G., Incorvaia, L., Flandina, C., Ancona, C., Fulfaro, F., Crescimanno, M., et al. (2016). Clinical impact of cystatin C/cathepsin L and follistatin/activin A systems in breast cancer progression: a preliminary report. Cancer Investig. 34, 415-423. doi: 10.1080/07357907.2016.1222416

Leto, G., Sepporta, M. V., Crescimanno, M., Flandina, C., and Tumminello, F. M. (2010). Cathepsin L in metastatic bone disease: therapeutic implications. Biol. Chem. 391, 655-664. doi: 10.1515/bc.2010.069

Lu, J., Wang, M., Wang, Z., Fu, Z., Lu, A., and Zhang, G. (2018). Advances in the discovery of cathepsin K inhibitors on bone resorption. J. Enzyme Inhibit. Med. Chem. 33, 890-904. doi: 10.1080/14756366.2018.1465417

Maciewicz, R. A., and Wotton, S. F. (1991). Degradation of cartilage matrix components by the cysteine proteinases, cathepsins B and L. Biomed. Biochim. Acta 50, 561-564.

Maciewicz, R. A., Wotton, S. F., Etherington, D. J., and Duance, V. C. (1990). Susceptibility of the cartilage collagens types II, IX and XI to degradation by the cysteine proteinases, cathepsins B and L. FEBS Lett. 269, 189-193. doi: 10.1016/0014-5793(90)81151-D

Mallari, J. P., Shelat, A., Kosinski, A., Caffrey, C. R., Connelly, M., Zhu, F., et al. (2008). Discovery of trypanocidal thiosemicarbazone inhibitors of rhodesain and TbcatB. Bioorgan. Med. Chem. Lett. 18, 2883-2885. doi: 10.1016/j.bmcl.2008.03.083

Mason, R. W., Gal, S., and Gottesman, M. M. (1987). The identification of the major excreted protein (MEP) from a transformed mouse fibroblast cell line as a catalytically active precursor form of cathepsin L. Biochem J. 248, 449-454. doi: 10.1042/bj2480449

Mason, R. W., Johnson, D. A., Barrett, A. J., and Chapman, H. A. (1986). Elastinolytic activity of human cathepsin L. Biochem. J. 233, 925-927. doi: 10.1042/bj2330925

Mason, R. W., and Massey, S. D. (1992). Surface activation of procathepsin L. Biochem. Biophys. Res. Commun. 189, 1659-1666. doi: 10.1016/0006-291X(92)90268-P

Mikhaylov, G., Klimpel, D., Schaschke, N., Mikac, U., Vizovisek, M., Fonovic, M., et al. (2014). Selective targeting of tumour and stromal cells by a nanocarrier system displaying lipidated cathepsin B inhibitor. Angew. Chem. Int. Edn. 53, 10077-10081. doi: 10.1002/anie.201402305

Nakagawa, T., Roth, W., Wong, P., Nelson, A., Farr, A., and Deussing, J., et al. (1998). Cathepsin L: critical role in Ii degradation and CD4 T cell selection in the thymus. Science 280, 450-453. doi: 10.1126/science.280.5362.450

Nguyen, Q., Mort, J. S., and Roughley, P. J. (1990). Cartilage proteoglycan aggregate is degraded more extensively by cathepsin L than by cathepsin B. Biochem. J. 266, 569-573. 
Nishida, Y., Kohno, K., Kawamata, T., Morimitsu, K., Kuwano, M., and Miyakawa, I. (1995). Increased cathepsin L levels in serum in some patients with ovarian cancer: comparison with CA125 and CA72-4. Gynecol Oncol. 56, 357-361. doi: 10.1006/gyno.1995.1063

Nishimura, Y., Furuno, K., and Kato, K. (1988). Biosynthesis and processing of lysosomal cathepsin L in primary cultures of rat hepatocytes. Arch. Biochem. Biophys. 263, 107-116. doi: 10.1016/0003-9861(88)90618-2

Nosaka, A. Y., Kanaori, K., Teno, N., Togame, H., Inaoka, T., and Takai, M., et al. (1999). Conformational studies on the specific cleavage site of Type I collagen (alpha-1) fragment (157-192) by cathepsins K and L by proton NMR spectroscopy. Bioorgan. Med. Chem. 7, 375-379. doi: 10.1016/S0968-0896(98)00227-2

Noureen, A., Jabeen, F., Tabish, T. A., Ali, M., Iqbal, R., Yaqub, S., et al. (2019). Histopathological changes and antioxidant responses in common carp (Cyprinus carpio) exposed to copper nanoparticles. Drug Chem. Toxicol. doi: 10.1080/01480545.2019.1606233. [Epub ahead of print].

Noureen, A., Jabeen, F., Tabish, T. A., Yaqub, S., Ali, M., and Chaudhry, A. S. (2018a). Assessment of copper nanoparticles (Cu-NPs) and copper (II) oxide $(\mathrm{CuO})$ induced hemato-and hepatotoxicity in Cyprinus carpio. Nanotechnology 29:144003. doi: 10.1088/1361-6528/aaaaa7

Noureen, A., Jabeen, F., Tabish, T. A., Zahoor, M. K., Ali, M., Iqbal, R., et al. (2018b). Ameliorative effects of Moringa oleifera on copper nanoparticle induced toxicity in Cyprinus carpio assessed by histology and oxidative stress markers. Nanotechnology 29:464003. doi: 10.1088/1361-6528/aade23

Nwosu, L. N., Gowler, P. R., Burston, J. J., Rizoska, B., Tunblad, K., Lindström, E., et al. (2018). Analgesic effects of the cathepsin K inhibitor L-006235 in the monosodium iodoacetate model of osteoarthritis pain. Pain Rep. 3:e685. doi: 10.1097/PR9.0000000000000685

Panwar, P., Law, S., Jamroz, A., Azizi, P., Zhang, D., Ciufolini, M., et al. (2018). Tanshinones that selectively block the collagenase activity of cathepsin $\mathrm{K}$ provide a novel class of ectosteric antiresorptive agents for bone. Brit. J. Pharmacol. 175, 902-923. doi: 10.1111/bph.14133

Parker, E. N., Odutola, S. O., Wang, Y., Strecker, T. E., Mukherjee, R., Shi, Z., et al. (2017). Synthesis and biological evaluation of a water-soluble phosphate prodrug salt and structural analogues of KGP94, a lead inhibitor of cathepsin L. Bioorgan. Med. Chem. Lett. 27, 1304-1310. doi: 10.1016/j.bmcl.2016.12.039

Parker, E. N., Song, J., Kumar, G. K., Odutola, S. O., Chavarria, G. E., Charlton-Sevcik, A. K., et al. (2015). Synthesis and biochemical evaluation of benzoylbenzophenone thiosemicarbazone analogues as potent and selective inhibitors of cathepsin L. Bioorgan. Med. Chem. 23, 6974-6992. doi: 10.1016/j.bmc.2015.09.036

Patra, J. K., Das, G., Fraceto, L. F., Campos, E. V. R., del Pilar RodriguezTorres, M., Acosta-Torres, L. S., et al. (2018). Nano based drug delivery systems: recent developments and future prospects. J. Nanobiotechnol. 16:71. doi: 10.1186/s12951-018-0392-8

Platell, C., Cooper, D., Papadimitriou, J. M., and Hall, J. C. (2000). The omentum. World J. Gastroenterol. 6, 169-176. doi: 10.3748/wjg.v6.i2.169

Potts, W., Bowyer, J., Jones, H., Tucker, D., Freemont, A. J., and Millest, A., et al. (2004). Cathepsin L-deficient mice exhibit abnormal skin and bone development and show increased resistance to osteoporosis following ovariectomy. Int. J. Exp. Pathol. 85, 85-96. doi: 10.1111/j.0959-9673.2004.00373.x

Pranjol, M. Z. I., Gutowski, N. J., Hannemann, M., and Whatmore, J. L. (2019a). Cathepsin L induces proangiogenic changes in human omental microvascular endothelial cells via activation of the ERK1/2 Pathway. Curr. Cancer Drug Targets 19, 231-242. doi: 10.2174/15680096186661808311 23951

Pranjol, M. Z. I., Zinovkin, D. A., Maskell, A. R. T., Stephens, L. J., Achinovich, S. L., and Los, D. M., et al. (2019b). Cathepsin L-induced galectin-1 may act as a proangiogenic factor in the metastasis of high-grade serous carcinoma. J. Transl. Med. 17, 216. doi: 10.1186/s12967-019-1963-7

Prence, E. M., Dong, J. M., and Sahagian, G. G. (1990). Modulation of the transport of a lysosomal enzyme by PDGF. J. Cell Biol. 110, 319-326. doi: $10.1083 /$ jcb.110.2.319

Qin, G., Cai, Y., Long, J., Zeng, H., Xu, W., and Li, Y., et al. (2016). Cathepsin L is involved in proliferation and invasion of breast cancer cells. Neoplasma 63, 30-36. doi: 10.4149/neo_2016_004
Renko, M., PoŽgan, U., Majera, D., and Turk, D. (2010). Stefin A displaces the occluding loop of cathepsin B only by as much as required to bind to the active site cleft. FEBS J. 277, 4338-4345. doi: 10.1111/j.1742-4658.2010.07824.x

Ritonja, A., Popovic, T., Kotnik, M., Machleidt, W., and Turk, V. (1988). Amino acid sequences of the human kidney cathepsins H and L. FEBS Lett. 228, 341-345. doi: 10.1016/0014-5793(88)80028-0

Ronkainen, N. J., and Okon, S. L. (2014). Nanomaterial-based electrochemical immunosensors for clinically significant biomarkers. Materials 7, 4669-4709. doi: $10.3390 / \operatorname{ma} 7064669$

Roth, W., Deussing, J., Botchkarev, V. A., Pauly-Evers, M., Saftig, P., and Hafner, A., et al. (2000). Cathepsin L deficiency as molecular defect of furless: hyperproliferation of keratinocytes and pertubation of hair follicle cycling. FASEB J. 14, 2075-2086. doi: 10.1096/fj.99-0970com

Rousselet, N., Mills, L., Jean, D., Tellez, C., Bar-Eli, M., and Frade, R. (2004). Inhibition of tumourigenicity and metastasis of human melanoma cells by anti-cathepsin L single chain variable fragment. Cancer Res. 64, 146-151. doi: 10.1158/0008-5472.CAN-03-1717

Samie, M. A., and Xu, H. (2014). Lysosomal exocytosis and lipid storage disorders. J. Lipid Res. 55, 995-1009. doi: 10.1194/jlr.R046896

Sée, V., Free, P., Cesbron, Y., Nativo, P., Shaheen, U., Rigden, D. J., et al. (2009). Cathepsin L digestion of nanobioconjugates upon endocytosis. ACS Nano 3, 2461-2468. doi: 10.1021/nn9006994

Shimada, N., Ohno-Matsui, K., Iseki, S., Koike, M., Uchiyama, Y., and Wang, J., et al. (2010). Cathepsin L in bone marrow-derived cells is required for retinal and choroidal neovascularization. Am. J. Pathol. 176, 2571-2580. doi: 10.2353/ajpath.2010.091027

Song, J., Jones, L. M., Chavarria, G. E., Charlton-Sevcik, A. K., Jantz, A., Johansen, A., et al. (2013). Small-molecule inhibitors of cathepsin L incorporating functionalized ring-fused molecular frameworks. Bioorgan. Med. Chem. Lett. 23, 2801-2807. doi: 10.1016/j.bmcl.2012.12.025

Speshock, J. L., Braydich-Stolle, L. K., Szymanski, E. R., and Hussain, S. M. (2011) Silver and gold nanoparticles alter cathepsin activity in vitro. Nanoscale Res. Lett. 6:17. doi: 10.1007/s11671-010-9746-3

Sudhan, D. R., Pampo, C., Rice, L., and Siemann, D. W. (2016). Cathepsin $\mathrm{L}$ inactivation leads to multimodal inhibition of prostate cancer cell dissemination in a preclinical bone metastasis model. Int. J. Cancer 138, 2665-2677. doi: 10.1002/ijc.29992

Sudhan, D. R., and Siemann, D. W. (2013). Cathepsin L inhibition by the small molecule KGP94 suppresses tumour microenvironment enhanced metastasis associated cell functions of prostate and breast cancer cells. Clin. Exp. Metast. 30, 891-902. doi: 10.1007/s10585-013-9590-9

Sudhan, D. R., and Siemann, D. W. (2015). Cathepsin L targeting in cancer treatment. Pharmacol. Therap. 155, 105-116. doi: 10.1016/j.pharmthera.2015.08.007

Sui, H., Shi, C., Yan, Z., and Wu, M. (2016). Overexpression of Cathepsin L is associated with chemoresistance and invasion of epithelial ovarian cancer. Oncotarget 7:45995. doi: 10.18632/oncotarget.10276

Tabish, T. A. (2018). Graphene-based materials: the missing piece in nanomedicine?. Biochem. Biophys. Res. Commun. 504, 686-689. doi: 10.1016/j.bbrc.2018.09.029

Tabish, T. A., Lin, L., Ali, M., Jabeen, F., Ali, M., Iqbal, R., et al. (2018a). Investigating the bioavailability of graphene quantum dots in lung tissues via Fourier transform infrared spectroscopy. Interface focus 8:20170054. doi: 10.1098/rsfs.2017.0054

Tabish, T. A., Pranjol, M. Z. I., Hayat, H., Rahat, A. A., Abdullah, T. M., Whatmore, J. L., et al. (2017). In vitro toxic effects of reduced graphene oxide nanosheets on lung cancer cells. Nanotechnology 28:504001. doi: 10.1088/1361-6528/aa95a8

Tabish, T. A., Pranjol, M. Z. I., Horsell, D. W., Rahat, A. A., Whatmore, J. L. Winyard, P. G., et al. (2019). Graphene oxide-based targeting of extracellular cathepsin D and cathepsin $\mathrm{L}$ as a novel anti-metastatic enzyme cancer therapy. Cancers 11:319. doi: 10.3390/cancers11030319

Tabish, T. A., Pranjol, M. Z. I., Jabeen, F., Abdullah, T., Latif, A., Khalid, A., et al. (2018b). Investigation into the toxic effects of graphene nanopores on lung cancer cells and biological tissues. Appl. Mater. Today 12, 389-401. doi: 10.1016/j.apmt.2018.07.005

Tabish, T. A., Scotton, C. J., J., Ferguson, D. C., Lin, L., der Veen, A. V., Lowry, S., et al. (2018c). Biocompatibility and toxicity of graphene quantum dots for 
potential application in photodynamic therapy. Nanomedicine 13, 1923-1937. doi: $10.2217 / \mathrm{nnm}-2018-0018$

Tabish, T. A., Zhang, S., and Winyard, P. G. (2018d). Developing the next generation of graphene-based platforms for cancer therapeutics: The potential role of reactive oxygen species. Redox Biol. 15, 34-40. doi: 10.1016/j.redox.2017.11.018

Tohda, C., and Tohda, M. (2017). Extracellular cathepsin L stimulates axonal growth in neurons. BMC Res. Notes. 10:613. doi: 10.1186/s13104-017-2940-y

Urbich, C., Heeschen, C., Aicher, A., Sasaki, K., Bruhl, T., and Farhadi, M. R., et al. (2005). Cathepsin $\mathrm{L}$ is required for endothelial progenitor cell-induced neovascularization. Nat. Med. 11, 206-213. doi: 10.1038/nm1182

van den Brûle, F., Califice, S., Garnier, F., Fernandez, P. L., Berchuck, A., and Castronovo, V. (2003). Galectin-1 accumulation in the ovary carcinoma peritumoural stroma is induced by ovary carcinoma cells and affects both cancer cell proliferation and adhesion to laminin-1 and fibronectin. Lab Invest. 83, 377-386. doi: 10.1097/01.LAB.0000059949.01480.40

von Figura, K., and Hasilik, A. (1986). Lysosomal enzymes and their receptors. Ann. Rev. Biochem. 55, 167-193. doi: 10.1146/annurev.bi.55.070186.001123

Winiarski, B. K., Cope, N., Alexander, M., Pilling, L. C., Warren, S., and Acheson, $\mathrm{N}$., et al. (2014). Clinical relevance of increased endothelial and mesothelial expression of proangiogenic proteases and VEGFA in the omentum of patients with metastatic ovarian high-grade serous carcinoma. Transl Oncol. 7, 267-76 e4. doi: 10.1016/j.tranon.2014.02.013

Winiarski, B. K., Wolanska, K. I., Rai, S., Ahmed, T., Acheson, N., and Gutowski, N. J., et al. (2013). Epithelial ovarian cancer-induced angiogenic phenotype of human omental microvascular endothelial cells may occur independently of VEGF signaling. Transl. Oncol. 6, 703-714. doi: 10.1593/tlo.13529

Withana, N. P., Blum, G., Sameni, M., Slaney, C., Anbalagan, A., Olive, M. B., et al. (2012). Cathepsin B inhibition limits bone metastasis in breast cancer. Cancer Res. 72, 1199-1209. doi: 10.1158/0008-5472.CAN-1 $1-2759$

Woo, J. T., Yamaguchi, K., Hayama, T., Kobori, T., Sigeizumi, S., and Sugimoto, K., et al. (1996). Suppressive effect of N-(benzyloxycarbonyl)-L-phenylalanylL-tyrosinal on bone resorption in vitro and in vivo. Eur. J. Pharmacol. 300, 131-135. doi: 10.1016/0014-2999(95)00858-6
Xu, X., Greenland, J., Baluk, P., Adams, A., Bose, O., and McDonald, D. M., et al. (2013). Cathepsin L protects mice from mycoplasmal infection and is essential for airway lymphangiogenesis. Am. J. Respir. Cell Mol. Biol. 49, 437-444. doi: $10.1165 / \mathrm{rcmb} .2013-0016 \mathrm{OC}$

Xue, S. T., Wang, Y. L., Han, X. W., Yi, H., Jiang, W., Si, S. Y., et al. (2019). Novel cathepsin $\mathrm{K}$ inhibitors block osteoclasts in vitro and increase spinal bone density in zebrafish. RSC Adv. 9, 8600-8607. doi: 10.1039/C8RA10338K

Yang, Z., and Cox, J. L. (2007). Cathepsin L increases invasion and migration of B16 melanoma. Cancer Cell Int. 7:8. doi: 10.1186/1475-2867-7-8

Yoneda, T., and Hiraga, T. (2005). Crosstalk between cancer cells and bone microenvironment in bone metastasis. Biochem. Biophys. Res. Commun. 328, 679-687. doi: 10.1016/j.bbrc.2004.11.070

Zhang, L., Wei, L., Shen, G., He, B., Gong, W., and Min, N., et al. (2015). Cathepsin $\mathrm{L}$ is involved in proliferation and invasion of ovarian cancer cells. Mol. Med. Rep. 11, 468-474. doi: 10.3892/mmr.2014.2706

Zhang, W., Fan, W., Ottemann, B. M., Alshehri, S., and Garrison, J. C. (2020). Development of improved tumour-residualizing, GRPR-targeted agents: preclinical comparison of an endolysosomal trapping approach in agonistic and antagonistic constructs. J. Nuclear Med. 61, 443-450. doi: 10.2967/jnumed.119.231282

Zhang, W., Wang, S., Wang, Q., Yang, Z., Pan, Z., and Li, L. (2014). Overexpression of cysteine cathepsin $\mathrm{L}$ is a marker of invasion and metastasis in ovarian cancer. Oncol Rep. 31, 1334-1342. doi: 10.3892/or.2014.2967

Conflict of Interest: The authors declare that the research was conducted in the absence of any commercial or financial relationships that could be construed as a potential conflict of interest.

Copyright $\odot 2020$ Tabish, Pranjol, Whatmore and Zhang. This is an open-access article distributed under the terms of the Creative Commons Attribution License (CC $B Y)$. The use, distribution or reproduction in other forums is permitted, provided the original author(s) and the copyright owner(s) are credited and that the original publication in this journal is cited, in accordance with accepted academic practice. No use, distribution or reproduction is permitted which does not comply with these terms. 